DESY 00-085

ISSN 0418-9833

June 2000

\title{
Inclusive Photoproduction of Neutral Pions in the Photon Hemisphere at HERA
}

\author{
H1 Collaboration
}

\begin{abstract}
The inclusive cross section for the photoproduction of neutral pions has been measured as a function of the transverse momentum, rapidity, and Feynman $x$ of the $\pi^{0}$ mesons at an average photon-proton centre-of-mass energy of $208 \mathrm{GeV}$ and for photon virtualities below $Q^{2}=0.01 \mathrm{GeV}^{2}$. The $\pi^{0}$ measurement extends the range covered by previous charged particle measurements at HERA by two units of rapidity in the photon direction down to a value of -5.5 in the $\gamma p$ centre-of-mass frame. The $\pi^{0}$ transverse momentum distribution is well described over the whole measured range by a power law ansatz, while an exponential fit falls below the data at transverse momentum values above $1.5 \mathrm{GeV} / \mathrm{c}$. Good agreement with the predictions of the Monte Carlo models PYTHIA and PHOJET is found. In the context of the PYTHIA model the data are inconsistent with large intrinsic transverse momentum values in the photon.
\end{abstract}

To be submitted to European Physics Journal 
C. Adloff ${ }^{33}$, V. Andreev ${ }^{24}$, B. Andrieu ${ }^{27}$, V. Arkadov ${ }^{35}$, A. Astvatsatourov ${ }^{35}$, I. Ayyaz ${ }^{28}$, A. Babaev $^{23}$, J. Bähr ${ }^{35}$, P. Baranov ${ }^{24}$, E. Barrelet ${ }^{28}$, W. Bartel ${ }^{10}$, U. Bassler ${ }^{28}$, P. Bate ${ }^{21}$, A. Beglarian ${ }^{34}$, O. Behnke ${ }^{10}$, C. Beier ${ }^{14}$, A. Belousov ${ }^{24}$, T. Benisch ${ }^{10}$, Ch. Berger ${ }^{1}$, G. Bernardi ${ }^{28}$, T. Berndt ${ }^{14}$, G. Bertrand-Coremans ${ }^{4}$, J.C. Bizot ${ }^{26}$, K. Borras ${ }^{7}$, V. Boudry ${ }^{27}$, W. Braunschweig ${ }^{1}$, V. Brisson ${ }^{26}$, H.-B. Bröker ${ }^{2}$, D.P. Brown ${ }^{21}$, W. Brückner ${ }^{12}$, P. Bruel ${ }^{27}$, D. Bruncko ${ }^{16}$, J. Bürger ${ }^{10}$, F.W. Büsser ${ }^{11}$, A. Bunyatyan ${ }^{12,34}$, H. Burkhardt ${ }^{14}$, A. Burrage ${ }^{18}$, G. Buschhorn ${ }^{25}$, A.J. Campbell ${ }^{10}$, J. Cao ${ }^{26}$, T. Carli ${ }^{25}$, S. Caron ${ }^{1}$, E. Chabert ${ }^{22}$, D. Clarke ${ }^{5}$, B. Clerbaux ${ }^{4}$, C. Collard ${ }^{4}$, J.G. Contreras ${ }^{7,41}$, J.A. Coughlan ${ }^{5}$, M.-C. Cousinou ${ }^{22}$, B.E. $\operatorname{Cox}^{21}$, G. Cozzika ${ }^{9}$, J. Cvach $^{29}$, J.B. Dainton ${ }^{18}$, W.D. Dau ${ }^{15}$, K. Daum ${ }^{33,39}$, M. David ${ }^{9, \dagger}$, M. Davidsson ${ }^{20}$, B. Delcourt ${ }^{26}$, N. Delerue ${ }^{22}$, R. Demirchyan ${ }^{34}$, A. De Roeck ${ }^{10,43}$, E.A. De Wolf ${ }^{4}$, C. Diaconu ${ }^{22}$, P. Dixon ${ }^{19}$, V. Dodonov ${ }^{12}$, K.T. Donovan ${ }^{19}$, J.D. Dowell ${ }^{3}$, A. Droutskoi ${ }^{23}$, C. Duprel ${ }^{2}$, J. Ebert ${ }^{33}$, G. Eckerlin ${ }^{10}$, D. Eckstein ${ }^{35}$, V. Efremenko ${ }^{23}$, S. Egli ${ }^{32}$, R. Eichler ${ }^{36}$, F. Eisele ${ }^{13}$, E. Eisenhandler ${ }^{19}$, M. Ellerbrock ${ }^{13}$, E. Elsen ${ }^{10}$, M. Erdmann ${ }^{10,40, e}$, P.J.W. Faulkner ${ }^{3}$, L. Favart ${ }^{4}$, A. Fedotov ${ }^{23}$, R. Felst ${ }^{10}$, J. Ferencei ${ }^{10}$, F. Ferrarotto ${ }^{31}$, S. Ferron ${ }^{27}$, M. Fleischer ${ }^{10}$, G. Flügge ${ }^{2}$, A. Fomenko ${ }^{24}$, I. Foresti ${ }^{37}$, J. Formánek ${ }^{30}$, J.M. Foster ${ }^{21}$, G. Franke ${ }^{10}$, E. Gabathuler ${ }^{18}$, K. Gabathuler ${ }^{32}$, J. Garvey ${ }^{3}$, J. Gassner ${ }^{32}$, J. Gayler ${ }^{10}$, R. Gerhards ${ }^{10}$, S. Ghazaryan ${ }^{34}$,

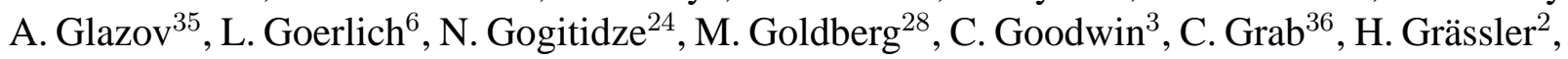
T. Greenshaw ${ }^{18}$, G. Grindhammer ${ }^{25}$, T. Hadig ${ }^{1}$, D. Haidt ${ }^{10}$, L. Hajduk ${ }^{6}$, V. Haustein ${ }^{33}$, W.J. Haynes ${ }^{5}$, B. Heinemann ${ }^{18}$, G. Heinzelmann ${ }^{11}$, R.C.W. Henderson ${ }^{17}$, S. Hengstmann ${ }^{37}$, H. Henschel ${ }^{35}$, R. Heremans ${ }^{4}$, G. Herrera ${ }^{7,41, k}$, I. Herynek ${ }^{29}$, M. Hilgers ${ }^{36}$, K.H. Hiller ${ }^{35}$, C.D. Hilton ${ }^{21}$, J. Hladký ${ }^{29}$, P. Höting ${ }^{2}$, D. Hoffmann ${ }^{10}$, W. Hoprich ${ }^{12}$, R. Horisberger ${ }^{32}$, S. Hurling ${ }^{10}$, M. Ibbotson ${ }^{21}$, Ç. İşsever ${ }^{7}$, M. Jacquet ${ }^{26}$, M. Jaffre ${ }^{26}$, L. Janauschek ${ }^{25}$, D.M. Jansen ${ }^{12}$, X. Janssen ${ }^{4}$, V. Jemanov ${ }^{11}$, L. Jönsson ${ }^{20}$, D.P. Johnson ${ }^{4}$, M.A.S. Jones ${ }^{18}$, H. Jung ${ }^{20}$, H.K. Kästli ${ }^{36}$, D. Kant ${ }^{19}$, M. Kapichine ${ }^{8}$, M. Karlsson ${ }^{20}$, O. Karschnick ${ }^{11}$, O. Kaufmann ${ }^{13}$, M. Kausch ${ }^{10}$, F. Keil ${ }^{14}$, N. Keller ${ }^{13}$, J. Kennedy ${ }^{18}$, I.R. Kenyon ${ }^{3}$, S. Kermiche ${ }^{22}$, C. Kiesling ${ }^{25}$, M. Klein ${ }^{35}$, C. Kleinwort ${ }^{10}$, G. Knies ${ }^{10}$, B. Koblitz ${ }^{25}$, H. Kolanoski ${ }^{38}$, S.D. Kolya ${ }^{21}$, V. Korbel ${ }^{10}$, P. Kostka ${ }^{35}$, S.K. Kotelnikov ${ }^{24}$, M.W. Krasny ${ }^{28}$, H. Krehbiel ${ }^{10}$, J. Kroseberg ${ }^{37}$, D. Krücker ${ }^{38}$, K. Krüger ${ }^{10}$, A. Küpper ${ }^{33}$, T. Kuhr ${ }^{11}$, T. Kurča ${ }^{35}$, R. Kutuev ${ }^{12}$, W. Lachnit ${ }^{10}$, R. Lahmann ${ }^{10}$, D. Lamb ${ }^{3}$, M.P.J. Landon ${ }^{19}$, W. Lange ${ }^{35}$, T. Laštovička ${ }^{30}$, A. Lebedev ${ }^{24}$, B. Leißner ${ }^{1}$, V. Lemaitre ${ }^{10}$, R. Lemrani ${ }^{10}$, V. Lendermann ${ }^{7}$, S. Levonian ${ }^{10}$, M. Lindstroem ${ }^{20}$, G. Lobo $^{26}$, E. Lobodzinska ${ }^{10,6}$, B. Lobodzinski ${ }^{6,10}$, N. Loktionova ${ }^{24}$, V. Lubimov ${ }^{23}$, S. Lüders ${ }^{36}$, D. Lüke ${ }^{7,10}$, L. Lytkin ${ }^{12}$, N. Magnussen ${ }^{33}$, H. Mahlke-Krüger ${ }^{10}$, N. Malden ${ }^{21}$, E. Malinovski ${ }^{24}$, I. Malinovski ${ }^{24}$, R. Maraček ${ }^{25}$, P. Marage ${ }^{4}$, J. Marks ${ }^{13}$, R. Marshall ${ }^{21}$, H.-U. Martyn ${ }^{1}$, J. Martyniak ${ }^{6}$, S.J. Maxfield ${ }^{18}$, A. Mehta ${ }^{18}$, K. Meier ${ }^{14}$, P. Merkel ${ }^{10}$, F. Metlica ${ }^{12}$, A. Meyer ${ }^{10}$, H. Meyer ${ }^{33}$, J. Meyer ${ }^{10}$, P.-O. Meyer ${ }^{2}$, S. Mikocki ${ }^{6}$, D. Milstead ${ }^{18}$, T. Mkrtchyan ${ }^{34}$, R. Mohr ${ }^{25}$, S. Mohrdieck ${ }^{11}$, M.N. Mondragon ${ }^{7}$, F. Moreau ${ }^{27}$, A. Morozov ${ }^{8}$, J.V. Morris ${ }^{5}$, D. Müller ${ }^{37}$, K. Müller ${ }^{13}$, P. Murín ${ }^{16,42}$, V. Nagovizin ${ }^{23}$, B. Naroska ${ }^{11}$, J. Naumann ${ }^{7}$, Th. Naumann ${ }^{35}$, I. Négri ${ }^{22}$, G. Nellen ${ }^{25}$, P.R. Newman ${ }^{3}$, T.C. Nicholls ${ }^{5}$, F. Niebergall ${ }^{11}$, C. Niebuhr ${ }^{10}$, O. Nix ${ }^{14}$, G. Nowak $^{6}$, T. Nunnemann ${ }^{12}$, J.E. Olsson ${ }^{10}$, D. Ozerov ${ }^{23}$, V. Panassik ${ }^{8}$, C. Pascaud $^{26}$, S. Passaggio ${ }^{36}$, G.D. Patel ${ }^{18}$, E. Perez ${ }^{9}$, J.P. Phillips ${ }^{18}$, D. Pitzl ${ }^{10}$, R. Pöschl ${ }^{7}$, I. Potachnikova ${ }^{12}$, B. Povh ${ }^{12}$, K. Rabbertz ${ }^{1}$, G. Rädel ${ }^{9}$, J. Rauschenberger ${ }^{11}$,

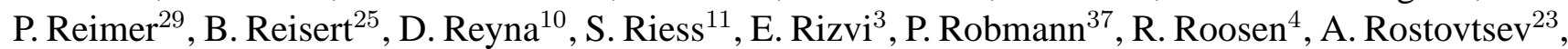
C. Royon ${ }^{9}$, S. Rusakov ${ }^{24}$, K. Rybicki ${ }^{6}$, D.P.C. Sankey ${ }^{5}$, J. Scheins ${ }^{1}$, F.-P. Schilling ${ }^{13}$, S. Schleif ${ }^{14}$, P. Schleper ${ }^{13}$, D. Schmidt ${ }^{33}$, D. Schmidt ${ }^{10}$, L. Schoeffel ${ }^{9}$, A. Schöning ${ }^{36}$, T. Schörner ${ }^{25}$, V. Schröder ${ }^{10}$, H.-C. Schultz-Coulon ${ }^{10}$, K. Sedlák ${ }^{29}$, F. Sefkow ${ }^{37}$, V. Shekelyan ${ }^{25}$, I. Sheviakov ${ }^{24}$, L.N. Shtarkov ${ }^{24}$, G. Siegmon ${ }^{15}$, P. Sievers ${ }^{13}$, Y. Sirois ${ }^{27}$, T. Sloan $^{17}$, P. Smirnov ${ }^{24}$, M. Smith ${ }^{18}$, V. Solochenko ${ }^{23}$, Y. Soloviev ${ }^{24}$, V. Spaskov ${ }^{8}$, A. Specka ${ }^{27}$, H. Spitzer ${ }^{11}$, R. Stamen ${ }^{7}$, J. Steinhart ${ }^{11}$, B. Stella ${ }^{31}$, A. Stellberger ${ }^{14}$, J. Stiewe ${ }^{14}$, U. Straumann ${ }^{37}$, W. Struczinski ${ }^{2}$, J.P. Sutton ${ }^{3}$, M. Swart ${ }^{14}$, M. Taševský ${ }^{29}$, V. Tchernyshov ${ }^{23}$, S. Tchetchelnitski ${ }^{23}$, G. Thompson ${ }^{19}$, P.D. Thompson ${ }^{3}$, N. Tobien ${ }^{10}$, D. Traynor ${ }^{19}$, 
P. Truöl ${ }^{37}$, G. Tsipolitis ${ }^{36}$, J. Turnau ${ }^{6}$, J.E. Turney ${ }^{19}$, E. Tzamariudaki ${ }^{25}$, S. Udluft ${ }^{25}$, A. Usik ${ }^{24}$, S. Valkár ${ }^{30}$, A. Valkárováa ${ }^{30}$, C. Vallée ${ }^{22}$, P. Van Mechelen ${ }^{4}$, Y. Vazdik ${ }^{24}$, S. von Dombrowski ${ }^{37}$, K. Wacker ${ }^{7}$, R. Wallny ${ }^{13}$, T. Walter ${ }^{37}$, B. Waugh ${ }^{21}$, G. Weber ${ }^{11}$, M. Weber ${ }^{14}$, D. Wegener ${ }^{7}$, A. Wegner ${ }^{11}$, T. Wengler ${ }^{13}$, M. Werner ${ }^{13}$, G. White ${ }^{17}$, S. Wiesand ${ }^{33}$, T. Wilksen ${ }^{10}$, M. Winde ${ }^{35}$, G.-G. Winter ${ }^{10}$, C. Wissing ${ }^{7}$, M. Wobisch ${ }^{2}$, H. Wollatz ${ }^{10}$, E. Wünsch ${ }^{10}$, J. Žáček ${ }^{30}$, J. Zálešák ${ }^{30}$, Z. Zhang ${ }^{26}$, A. Zhokin ${ }^{23}$, F. Zomer ${ }^{26}$, J. Zsembery ${ }^{9}$ and M. zur Nedden ${ }^{10}$

${ }^{1}$ I. Physikalisches Institut der RWTH, Aachen, Germany ${ }^{a}$

${ }^{2}$ III. Physikalisches Institut der RWTH, Aachen, Germany ${ }^{a}$

${ }^{3}$ School of Physics and Space Research, University of Birmingham, Birmingham, UK ${ }^{b}$

${ }^{4}$ Inter-University Institute for High Energies ULB-VUB, Brussels; Universitaire Instelling Antwerpen, Wilrijk; Belgium ${ }^{c}$

${ }^{5}$ Rutherford Appleton Laboratory, Chilton, Didcot, $\mathrm{UK}^{b}$

${ }^{6}$ Institute for Nuclear Physics, Cracow, Poland ${ }^{d}$

${ }^{7}$ Institut für Physik, Universität Dortmund, Dortmund, Germany ${ }^{a}$

8 Joint Institute for Nuclear Research, Dubna, Russia

${ }^{9}$ DSM/DAPNIA, CEA/Saclay, Gif-sur-Yvette, France

${ }^{10}$ DESY, Hamburg, Germany ${ }^{a}$

${ }^{11}$ II. Institut für Experimentalphysik, Universität Hamburg, Hamburg, Germany ${ }^{a}$

${ }^{12}$ Max-Planck-Institut für Kernphysik, Heidelberg, Germany ${ }^{a}$

${ }^{13}$ Physikalisches Institut, Universität Heidelberg, Heidelberg, Germany ${ }^{a}$

${ }^{14}$ Kirchhoff-Institut für Physik, Universität Heidelberg, Heidelberg, Germany ${ }^{a}$

${ }^{15}$ Institut für experimentelle und angewandte Physik, Universität Kiel, Kiel, Germany ${ }^{a}$

${ }^{16}$ Institute of Experimental Physics, Slovak Academy of Sciences, Košice, Slovak Republic ${ }^{e, f}$

${ }^{17}$ School of Physics and Chemistry, University of Lancaster, Lancaster, $\mathrm{UK}^{b}$

${ }^{18}$ Department of Physics, University of Liverpool, Liverpool, UK $^{b}$

${ }^{19}$ Queen Mary and Westfield College, London, $\mathrm{UK}^{b}$

${ }^{20}$ Physics Department, University of Lund, Lund, Sweden ${ }^{g}$

${ }^{21}$ Department of Physics and Astronomy, University of Manchester, Manchester, UK ${ }^{b}$

${ }^{22}$ CPPM, CNRS/IN2P3 - Univ Mediterranee, Marseille - France

${ }^{23}$ Institute for Theoretical and Experimental Physics, Moscow, Russia

${ }^{24}$ Lebedev Physical Institute, Moscow, Russia ${ }^{e, h}$

${ }^{25}$ Max-Planck-Institut für Physik, München, Germany ${ }^{a}$

${ }^{26}$ LAL, Université de Paris-Sud, IN2P3-CNRS, Orsay, France

${ }^{27}$ LPNHE, École Polytechnique, IN2P3-CNRS, Palaiseau, France

${ }^{28}$ LPNHE, Universités Paris VI and VII, IN2P3-CNRS, Paris, France

${ }^{29}$ Institute of Physics, Academy of Sciences of the Czech Republic, Praha, Czech Republic ${ }^{e, i}$

${ }^{30}$ Faculty of Mathematics and Physics, Charles University, Praha, Czech Republic ${ }^{e, i}$

${ }^{31}$ INFN Roma 1 and Dipartimento di Fisica, Università Roma 3, Roma, Italy

${ }^{32}$ Paul Scherrer Institut, Villigen, Switzerland

${ }^{33}$ Fachbereich Physik, Bergische Universität Gesamthochschule Wuppertal, Wuppertal, Germany ${ }^{a}$

${ }^{34}$ Yerevan Physics Institute, Yerevan, Armenia

${ }^{35}$ DESY, Zeuthen, Germany ${ }^{a}$

${ }^{36}$ Institut für Teilchenphysik, ETH, Zürich, Switzerland ${ }^{j}$

${ }^{37}$ Physik-Institut der Universität Zürich, Zürich, Switzerland ${ }^{j}$

${ }^{38}$ Present address: Institut für Physik, Humboldt-Universität, Berlin, Germany

${ }^{39}$ Also at Rechenzentrum, Bergische Universität Gesamthochschule Wuppertal, Wuppertal, 
Germany

${ }^{40}$ Also at Institut für Experimentelle Kernphysik, Universität Karlsruhe, Karlsruhe, Germany

${ }^{41}$ Also at Dept. Fis. Ap. CINVESTAV, Mérida, Yucatán, México ${ }^{k}$

${ }^{42}$ Also at University of P.J. Šafárik, Košice, Slovak Republic

${ }^{43}$ Also at CERN, Geneva, Switzerland

${ }^{a}$ Supported by the Bundesministerium für Bildung, Wissenschaft, Forschung und Technologie, FRG, under contract numbers 7AC17P, 7AC47P, 7DO55P, 7HH17I, 7HH27P, 7HD17P, 7HD27P, 7KI17I, 6MP17I and 7WT87P

${ }^{b}$ Supported by the UK Particle Physics and Astronomy Research Council, and formerly by the UK Science and Engineering Research Council

${ }^{c}$ Supported by FNRS-FWO, IISN-IIKW

${ }^{d}$ Partially Supported by the Polish State Committee for Scientific Research, grant No. 2P0310318 and SPUB/DESY/P-03/DZ 1/99

${ }^{e}$ Supported by the Deutsche Forschungsgemeinschaft

${ }^{f}$ Supported by VEGA SR grant no. 2/5167/98

${ }^{g}$ Supported by the Swedish Natural Science Research Council

${ }^{h}$ Supported by Russian Foundation for Basic Research grant no. 96-02-00019

${ }^{i}$ Supported by GA AVČR grant number no. A1010821

${ }^{j}$ Supported by the Swiss National Science Foundation

${ }^{k}$ Supported by CONACyT 


\section{Introduction}

At the electron-proton collider HERA, photoproduction $(\gamma p)$ processes are induced by quasireal photons that are emitted by the incoming electron or positron'. The majority of the photonproton interactions are "soft" scattering processes in which particles with limited transverse momenta are produced. A small fraction of $\gamma p$ processes, however, involve "hard" scatterings. In these, the photon interacts either as a single object with a parton from the proton ("direct" photon interactions) or via a parton-parton scattering after fluctuating into a partonic system. These latter "resolved" photon processes give rise to a photon remnant following the direction of the incoming photon.

In the study of photoproduction scattering dynamics inclusive spectra of identified particles constitute an important source of information. Investigations of inclusive spectra using charged particles at HERA have confirmed the general features of hard photon-proton scattering outlined above. In particular it was shown that in these photoproduction processes there is an excess of charged particles in the region of large transverse momenta, as compared to hadronhadron scattering processes, and that this excess is in agreement with perturbative Quantum Chromodynamics (QCD) calculations, which take into account both the direct processes and the hard components of the resolved photon processes. These studies were carried out using the central tracking detectors of the experiments H1 [1, 2] and ZEUS [3]. The photon hemisphere was explored for charged particles down to -1.5 units of pseudorapidity $\eta$ in the laboratory system[ and with transverse momenta up to $12 \mathrm{GeV} / \mathrm{c}$.

The $\pi^{0}$ measurement described here is an extension of the previous charged particle studies into an as yet unexplored phase space region in which the photon remnant is expected to dominate the particle flow. In this region charged particles could not be reliably reconstructed, because of limitations in acceptance of the H1 tracking devices. Thus, the present $\pi^{0}$ measurement covers two additional units of rapidity $y$, down to $y=-3.5$. We note that, since at HERA the $\gamma p$ centre-of-mass (CM) frame itself moves with an average rapidity of roughly two units relative to the laboratory system, this limit corresponds to $y_{\gamma p} \simeq-5.5$, as seen in the $\gamma p \mathrm{CM}$ frame.

The production of neutral pions has previously been extensively studied in several fixed target experiments [ 4 , 5], using both charged particle beams and photon beams, as well as in $p p$ and $p \bar{p}$ collider experiments [6]. The CM energies in these photoproduction experiments range up to $18 \mathrm{GeV}$. In this paper we study, for the first time at HERA, neutral pion photoproduction at $\gamma p \mathrm{CM}$ energies around $208 \mathrm{GeV}$. We measure the inclusive double differential $\pi^{0}$ photoproduction cross section as a function of the $\pi^{0}$ transverse momentum and rapidity in the laboratory frame. The $\pi^{0}$ mesons are identified through their decay into two photons which are detected in the electromagnetic calorimeters of the $\mathrm{H} 1$ detector.

The paper is organized as follows. In section 2, we give a short description of the H1 detector components that are most relevant to the $\pi^{0}$ cross section measurement. In section 3 , we

\footnotetext{
${ }^{1}$ In the running periods used for this analysis, HERA was operated with a positron beam.

${ }^{2}$ The pseudorapidity and rapidity are defined as $\eta=-\ln \tan (\theta / 2)$ and $y=\frac{1}{2} \ln \frac{E+p_{z}}{E-p_{z}}$, respectively, where $E$ is the particle energy, $p_{z}$ the momentum component with respect to the $z$-axis and $\theta$ the polar angle; the $z$-axis is given by the proton direction.
} 
describe the Monte Carlo models used to correct the data. Section 4 outlines the data selection and the analysis method, while the systematic errors are discussed in section 5. The results are presented and discussed in section 6. Finally, a summary is given in section 7.

\section{Detector Description}

A comprehensive description of the $\mathrm{H} 1$ detector is given in Ref. [7]. The parts of the detector vital for this analysis are the backward calorimeter (SpaCal), the Liquid Argon calorimeter (LAr), the central trackers and the electron and photon detectors of the luminosity system. Their main properties are described here.

The SpaCal [8] is a lead-scintillating fibre calorimeter separated into an electromagnetic (EM) and a hadronic section of equal size. The EM section consists of 1192 cells with a cross section of $40.5 \times 40.5 \mathrm{~mm}^{2}$, each read out by a photomultiplier tube. The hadronic section consists of 128 cells with a cross section of $120 \times 120 \mathrm{~mm}^{2}$. The EM section has a depth of 27 radiation lengths. The polar angle range covered by the SpaCal is $153^{\circ} \leq \theta \leq 178^{\circ}$.

The energy resolution of the EM SpaCal section for electromagnetically interacting particles is

$$
\frac{\sigma(E)}{E} \approx \frac{0.075}{\sqrt{E / \mathrm{GeV}}} \oplus 0.010
$$

where $\mathrm{E}$ is the energy deposited in the SpaCal. The absolute energy scale, in the lower energy range from $0.2 \mathrm{GeV}$ to $10 \mathrm{GeV}$, is known with an uncertainty of $4 \%$ [9]. The resolution in $\theta$ is better than $2.5 \mathrm{mrad}$ for energies above $1 \mathrm{GeV}$.

The LAr calorimeter [10] is highly segmented and consists of an electromagnetic section with lead absorbers, corresponding to a depth varying between 20 and 30 radiation lengths, and a hadronic section with steel absorbers. The resolution of the LAr EM section is

$$
\frac{\sigma(E)}{E} \approx \frac{0.12}{\sqrt{E / \mathrm{GeV}}} \oplus 0.01,
$$

as measured in test beams [11]. Furthermore the absolute energy scale of the LAr calorimeter, in the low energy range from $0.8 \mathrm{GeV}$ to $10 \mathrm{GeV}$, is known to a precision of $4 \%$. The LAr calorimeter covers the polar angle range $4^{\circ} \leq \theta \leq 154^{\circ}$.

The H1 central tracking system [7] is mounted concentrically around the beam line and covers polar angles in the range $20^{\circ}<\theta<160^{\circ}$. Measurements of the momenta of charged particles are provided by two coaxial cylindrical drift chambers (central jet chambers, CJC), mounted inside a homogeneous magnetic field of 1.15 Tesla. Further drift chambers are placed radially inside and outside the inner CJC chamber, providing accurate measurements of the $z$ coordinates of charged tracks. Finally, multiwire proportional chambers (MWPC), which allow triggering on those tracks, are also located within and between the CJC chambers. In the present analysis tracking information is used to identify isolated electromagnetic clusters in the LAr calorimeter, and to find the interaction vertices of the selected events. 
To detect photoproduction events, the electron and photon detectors of the H1 luminosity system [7] are used. They consist of $\mathrm{TlCl} / \mathrm{TlBr}$ crystal calorimeters with an energy resolution of

$\sigma(E) / E=0.22 / \sqrt{E / \mathrm{GeV}}$ and are located at $z=-33 \mathrm{~m}$ and $z=-103 \mathrm{~m}$, respectively. The detectors serve to identify scattered positrons from photoproduction processes. The signature of photoproduction is a signal in the electron detector with no accompanying signal in the photon detector.

\section{Event Simulation}

In this analysis, photoproduction interactions are modelled by two Monte Carlo generators, PHOJET (version 1.04) [12] and PYTHIA (version 5.722) [13]. These have both been shown to describe photoproduction data in previous analyses [1, 2, 14]. Both generators have in common that they use leading order (LO) QCD matrix elements for the hard scattering subprocess. Initial and final state parton radiation and the LUND fragmentation model [15] for hadronization are included, as implemented in the JETSET program [16]. The programs differ, however, in the treatment of multiple interactions and the transition from hard to soft processes at low transverse parton momentum.

The PHOJET event generator simulates all components that contribute to the total photoproduction cross section. It is based on the two-component dual parton model (for a review, see [17]). PHOJET incorporates simulations of soft processes on the basis of Regge theory. Soft and hard processes are connected through a unitarization scheme which serves to keep cross sections finite.

The PYTHIA event generator uses LO QCD calculations for the primary parton-parton scattering process and for multiple parton interactions. The latter are considered to arise from the scattering of partons from the photon and proton remnants. PYTHIA also models both hard and soft hadronic interactions - the latter by the exchange of low energy gluons - applying a unitarization scheme [18].

Additional transverse momentum is generated in PYTHIA by virtue of the assumed intrinsic (primordial) transverse momentum $\left(k_{\perp}\right)$ of the partons in the interacting hadrons. The $k_{\perp}$ distribution of the quark and antiquark from the photon, fluctuating into a hadronic state, is parametrized as a power law function $1 /\left(k_{\perp 0}^{2}+k_{\perp}^{2}\right)$ [18] with the value $k_{\perp 0}=400 \mathrm{MeV} / \mathrm{c}$. For this function PYTHIA applies for the primordial $k_{\perp}$ a cutoff, the value of which is taken from the transverse momentum of the individual hard scattering subprocess. PYTHIA offers alternative parametrizations for the $k_{\perp}$ distribution, by either a Gaussian or an exponential function. The predictions of the model for different $k_{\perp}$ distributions are compared with the experimental results in section 6 .

The PHOJET 1.04 event generator has no explicit intrinsic transverse momentum for partons entering a hard scattering.

For both Monte Carlo models the factorization and renormalization scales were set to the transverse momentum of the final state partons. GRV-LO [19] parton distribution functions for the proton and photon were used for the generation of events. 


\section{Data Selection and Analysis}

Photoproduction events were selected, which have the scattered positron registered in the electron detector of the luminosity system and consequently have a four-momentum transfer $Q^{2}<$ $10^{-2} \mathrm{GeV}^{2}$, where $Q^{2}=4 E_{e} E_{e}^{\prime} \cos ^{2}(\theta / 2)$. Here $E_{e}$ and $E_{e}^{\prime}$ are the incoming and scattered positron energies, and $\theta$ the polar angle of the scattered positron, $\theta \sim 180^{\circ}$. More specifically, the events were required to have a positron candidate registered in the fiducial volume of the electron detector, with energy $E_{e}^{\prime}>4 \mathrm{GeV}$, and to have less than $2 \mathrm{GeV}$ deposited in the photon detector. The latter condition suppresses background from the proton beam appearing in coincidence with the high rate of events from the Bethe-Heitler reaction $e p \rightarrow e \gamma p$. In order to ensure full efficiency and acceptance of the photoproduction tagging condition, the range of the variable $y_{\mathrm{B}}=1-\left(E_{e}^{\prime} / E_{e}\right)$ is restricted to $0.35<y_{\mathrm{B}}<0.65$ in the present analysis.

Two data samples were used in the analysis:

- The "minimum bias" data sample, collected in 1997 and corresponding to an integrated luminosity of $\sim 300 \mathrm{nb}^{-1}$. In addition to the photoproduction tagging condition, the trigger demanded only loose conditions on the presence of charged tracks in the central tracking detectors.

- The "SpaCal" sample, collected in 1996 and corresponding to an integrated luminosity of $\sim 4.3 \mathrm{pb}^{-1}$. The trigger used in this sample contained, in addition to the photoproduction tagging condition, requirements on the energy registered in the EM SpaCal. Thus at least one SpaCal energy cluster (i.e. an isolated energy deposition, separated from neighbouring energy depositions by the clustering algorithm) had to exceed $2 \mathrm{GeV}$, and at the same time have a radial distance to the beam axis of more than $16 \mathrm{~cm}$. In the subsequent analysis a trigger efficiency of at least $40 \%$ was ensured by demanding that in each event the most energetic SpaCal cluster had an energy exceeding $2.2 \mathrm{GeV}$ and a radial distance to the beam axis exceeding $16 \mathrm{~cm}$. Full trigger efficiency was reached for cluster energies and radial distances larger than $2.6 \mathrm{GeV}$ and $22 \mathrm{~cm}$, respectively.

The "SpaCal" sample allows the study of the phase space region in which the number of events in the "minimum bias" sample is too small, i.e. the region where the $\pi^{0}$ has both large negative rapidity and large transverse momentum.

In the further event selection the $z$-coordinate $z_{v}$ of the event interaction vertex, reconstructed using the central tracking detectors, had to satisfy the condition $\left|z_{v}\right|<35 \mathrm{~cm}$. The cut on $z_{v}$ suppresses beam-gas background events.

The resulting "minimum bias" and "SpaCal" data samples consist of $\sim 115 \mathrm{~K}$ events and $\sim 500 \mathrm{~K}$ events, respectively.

In the subsequent $\pi^{0}$ reconstruction, additional conditions were applied in order to accept energy clusters in the LAr and SpaCal EM calorimeters as photon candidates.

- For a LAr cluster to be accepted as a single photon candidate, the transverse radius of the cluster had to be smaller than $8 \mathrm{~cm}$ and the longitudinal cluster extension below $10 \mathrm{~cm}$. The cluster energy had to exceed $0.3 \mathrm{GeV}$. Furthermore, the distance between the cluster position and the impact point of the closest reconstructed charged track had to be larger than $5 \mathrm{~cm}$. 
- An EM SpaCal cluster was associated with a photon if it was reconstructed as an isolated energy deposition covering at least two SpaCal cells. The cluster energy had to exceed $0.3 \mathrm{GeV}$. A radial distance between $8 \mathrm{~cm}$ and $75 \mathrm{~cm}$ from the cluster to the beam axis was required to ensure full energy containment. Information from the backward tracking detectors was not used in selecting photon candidates in the SpaCal, since many photons shower in the material in front of the backward tracking detectors and the SpaCal.

The procedure for reconstructing and counting $\pi^{0}$ mesons was as follows: Each photon pair, from either the LAr or the SpaCal and satisfying the above criteria, was labelled according to transverse momentum $p_{\perp}$ and rapidity $y$. Photon four-momenta were derived from the cluster energy, the cluster coordinates and the coordinates of the interaction vertex. For every photon pair, the invariant mass $m_{\gamma \gamma}$ was calculated according to

$$
m_{\gamma \gamma}^{2} c^{4}=2 E_{1} E_{2}\left(1-\cos \psi_{12}\right) \text {. }
$$

Here $E_{1}$ and $E_{2}$ are the energies of the measured calorimeter clusters, attributed to the decay photons, and $\psi_{12}$ is their opening angle. Fig. 11 shows two-photon invariant mass distributions in several regions of transverse momentum $p_{\perp}$ and rapidity $y$ of photon pairs measured in the SpaCal (Fig. 1 a and 1 b), and in the LAr calorimeter (Fig. 1 c and 11d). These variables were calculated from the four-momentum sum of the two photons.

In each bin of $p_{\perp}$ and $y$ the two-photon mass distribution can be satisfactorily described by the sum of a Gaussian function for the $\pi^{0}$ signal and a background curve. The background was determined by fitting a polynomial of fourth order multiplied by a function of the type $\left(m_{\gamma \gamma}-m_{\gamma \gamma}^{t h r}\right)^{\alpha}$ in order to improve the threshold description where the background rises steeply. $m_{\gamma \gamma}^{t h r}$ is given by the centre of the lowest non-empty interval in the mass distribution, and $\alpha$ is a fitted parameter with $0 \leq \alpha \leq 1$.

The double differential cross section for inclusive $\pi^{0}$ photoproduction is given by the expression

$$
\frac{d^{2} \sigma_{\gamma p}}{d p_{\perp}^{2} d y}=\frac{N_{\text {prod }}\left(\Delta p_{\perp}, \Delta y\right)}{2 p_{\perp} \cdot \Delta p_{\perp} \cdot \Delta y \cdot \Phi \cdot \mathcal{L}}
$$

$N_{\text {prod }}$ is the number of $\pi^{0}$ s produced in the transverse momentum $\left(\Delta p_{\perp}\right)$ and rapidity $(\Delta y)$ interval considered, determined with all efficiency and acceptance corrections. The efficiency was determined by simulation of photoproduction events using the PHOJET and PYTHIA generators (see section 3 ), with full simulation of the detector response. $\mathcal{L}$ is the integrated luminosity, and $\Phi=0.00968$ is the photon flux factor, calculated according to the Weizsäcker - Williams formula [20] for the $y_{\mathrm{B}}$ range covered in this analysis. This range corresponds to the $\gamma p \mathrm{CM}$ energy range $177<\sqrt{s_{\gamma p}}<242 \mathrm{GeV}$, with an average of $208 \mathrm{GeV}$. We note that in the various bins of $p_{\perp}$ and $y$ the average $\sqrt{s_{\gamma p}}$ may deviate from this value by up to $\pm 3 \%$.

\section{Systematic Errors}

The errors on the results are dominated by systematic effects with the following main sources: 
1) The uncertainty of the fitting procedure arising from the determination of the combinatorial background in the fits of the two-photon mass distributions. In all kinematical intervals relevant for this analysis this was estimated by fitting these distributions, varying the fit parameters and fit ranges several times, following a random choice. Also the order of the polynomial used to describe the background was varied. The errors derived in this way vary substantially due to the statistical uncertainty of the background estimation. In order to get a conservative but reliable estimate for the uncertainty from the fitting procedure, a global error of $8 \%$ was therefore assigned, based on the distribution of the errors.

2) The $\pi^{0}$ reconstruction efficiencies, derived using the PYTHIA and PHOJET models, are in general in good agreement, except in the bins of high $p_{\perp}$ where the two models differ by up to $30 \%$. Such differences can be understood as being due to internal differences of the models in the population of certain kinematical regions. In the cross section calculations we have taken the mean reconstruction efficiency and assigned the difference as a bin to bin systematic error, varying between $1 \%$ and $15 \%$.

3) The uncertainty of the energy scales for the LAr and SpaCal calorimeters: Shifting the scale upward (downward) by $4 \%$ increases (reduces) the cross sections by about $20 \%$ for the data selected with the SpaCal trigger, and by about $10 \%$ for the minimum bias triggered data.

4) The uncertainty on the electron detector acceptance is $4 \%$ and 6\%, for the 1996 and 1997 data samples, respectively.

5) The uncertainty on the efficiency of the SpaCal trigger is found to be $1 \%$, from cross checks with an independent trigger.

6) The contribution of $\pi^{0} \mathrm{~s}$ from beam-gas interactions has been estimated to be less than $1 \%$ using the so-called "pilot bunches", i.e. positron beam bunches without partners with which they can collide in the proton beam.

7) The integrated luminosity is known to a precision of better than $2 \%$.

\section{Results}

The cross section for inclusive neutral pion production in $\gamma p$ interactions is displayed in Fig. 2 as a function of the $\pi^{0}$ rapidity for four intervals of the transverse momentum and in Fig. 3 as a function of $p_{\perp}$ for five intervals of rapidity. The data are summarized in Table 1. Rapidity is measured in the laboratory frame. Bin-centre corrections have been applied and errors are quoted as the sum of statistical and systematic errors, added in quadrature. In regions of low statistics (low $y$, high $p_{\perp}$ ) or large combinatorial background (central region, low $p_{\perp}$ ) unstable fits prevent the determination of cross section values.

Values derived from the $\mathrm{H} 1$ charged particle cross section measurements [1]], which cover the pseudorapidity range $|\eta| \leq 1.5$, are also shown (full triangles) in Figs. 2 and 3 . In order to compare these measurements with the $\pi^{0}$ result, the charged particle cross sections were first corrected for the non-pion part by subtracting a fraction of $(17.5 \pm 0.2) \%$ as derived from the PYTHIA and PHOJET predictions (the error reflects the difference in the predictions). The 
resulting $\pi^{+}+\pi^{-}$cross sections were then further divided by the isospin factor 2 . The $e p$ cross sections given in [1] have been converted into $\gamma p$ cross sections using the appropriate flux factor. While the charged pion cross section entries in Fig. 3 correspond directly to the original data points in [1], a smooth interpolation was used to arrive at the charged pion cross sections in Fig. 2, in order to account for the different transverse momentum ranges. The agreement between the neutral and charged pion cross sections is good in the kinematical range covered by both analyses. Figs. 2 and 3 demonstrate well the additional phase space region accessed with the present neutral pion analysis.

The model predictions according to the PHOJET and PYTHIA simulations are also shown in Figs. 2 and 3, with satisfactory agreement especially for PYTHIA. PHOJET predicts slightly too large a $\pi^{0}$ rate at high $p_{\perp}$ and low $y$.

The $\pi^{0}$ transverse momentum spectrum, integrated over the laboratory rapidity range covered by the SpaCal, i.e. $-3.5 \leq y \leq-1.5$, is shown in Fig. 4 . The data are summarized in Table 2. In deriving these cross sections also those regions were included for which no results are given in Table 1.

Transverse momentum spectra in high energy hadron - hadron collisions are successfully described by a power law ansatz of the form

$$
\frac{d^{2} \sigma}{d p_{\perp}^{2} d y}=A \cdot\left(1+p_{\perp} / p_{\perp 0}\right)^{-n}
$$

This QCD inspired ansatz [21, 22] was designed to describe transverse momentum spectra of centrally produced particles. It was shown in [1, 2, 3] that it fits well the H1 and ZEUS spectra measured for charged particles in the central pseudorapidity range. This is in agreement with observations made in many hadron-hadron experiments, see e.g. Kourkoumelis et al. [6] for centrally produced $\pi^{0} \mathrm{~s}$ at various centre-of-mass energies at the CERN ISR, and Banner et al. [6] for measurements performed at $\sqrt{s}=540 \mathrm{GeV}$ at the CERN SPS. A review of large $p_{\perp}$ particle production at the ISR is given by Geist et al. [23].

\begin{tabular}{|c||c|c|c|c|}
\hline \multicolumn{1}{|c||}{$y$} & \multicolumn{4}{c|}{$p_{\perp}[\mathrm{GeV} / \mathrm{c}]$} \\
\cline { 2 - 5 } & {$[0.2,0.6]$} & {$[0.6,0.8]$} & {$[0.8,1.0]$} & {$[1.0,2.0]$} \\
$(0.36)$ & $(0.69)$ & $(0.89)$ & $(1.31)$ \\
\hline \hline$[-3.5,-2.8](-3.15)$ & $47 \pm 10$ & $5.0 \pm 1.2$ & - & - \\
\hline$[-2.8,-2.4](-2.6)$ & $94 \pm 16$ & $12 \pm 2$ & $3.6 \pm 0.8$ & $0.23 \pm 0.08$ \\
\hline$[-2.4,-2.0](-2.2)$ & $158 \pm 27$ & $27 \pm 5$ & $8.0 \pm 2.0$ & $0.68 \pm 0.17$ \\
\hline$[-2.0,-1.5](-1.75)$ & $176 \pm 27$ & $28 \pm 5$ & $12.9 \pm 3.4$ & $1.34 \pm 0.32$ \\
\hline$[-0.5,+1.0](0.25)$ & - & - & $16.9 \pm 3.5$ & $1.97 \pm 0.35$ \\
\hline
\end{tabular}

Table 1: Inclusive $\pi^{0}$ photoproduction cross sections $d^{2} \sigma_{\gamma p} / d p_{\perp}^{2} d y\left(\mu \mathrm{b} /(\mathrm{GeV} / c)^{2}\right)$ for bins in the $\pi^{0}$ transverse momentum $p_{\perp}$ and rapidity $y$. The bin centres are given in addition to the interval limits. 


\begin{tabular}{|c||c|c|c|c|c|c|}
\hline \multicolumn{1}{|c||}{} & \multicolumn{6}{c|}{$p_{\perp}[\mathrm{GeV} / \mathrm{c}]$} \\
\cline { 2 - 7 }$y$ & {$[0.2,0.6]$} & {$[0.6,0.9]$} & {$[0.9,1.2]$} & {$[1.2,1.5]$} & {$[1.5,1.8]$} & {$[1.8,2.1]$} \\
& $(0.34)$ & $(0.73)$ & $(1.03)$ & $(1.33)$ & $(1.63)$ & $(1.93)$ \\
\hline \hline$[-3.5,-1.5]$ & $114 \pm 20$ & $12.5 \pm 2.4$ & $2.0 \pm 0.4$ & $0.47 \pm 0.15$ & $0.14 \pm 0.05$ & $0.04 \pm 0.01$ \\
\hline
\end{tabular}

Table 2: Inclusive $\pi^{0}$ photoproduction cross sections $d^{2} \sigma_{\gamma p} / d p_{\perp}^{2} d y\left(\mu \mathrm{b} /(\mathrm{GeV} / c)^{2}\right)$ for bins in the $\pi^{0}$ transverse momentum $p_{\perp}$ and for the rapidity range $-3.5 \leq y \leq-1.5$. The bin centres are given in addition to the interval limits.

The measurements presented in Fig. $¥$ have been performed in the backward region at large negative rapidities where phase space effects begin to be visible, causing a damping of transverse momentum spectra. This leads to a reduction of the cross section and to a steepening of the $p_{\perp}$ distribution. The power law ansatz does, nevertheless, satisfactorily describe the shape of the spectrum if the parameter $p_{\perp 0}$ is fixed to the value found in [1], namely $p_{\perp 0}=0.63 \mathrm{GeV} / \mathrm{c}$. A fit with the power law ansatz yields $n=8.0 \pm 0.2\left(\chi^{2} / \mathrm{ndf}=0.85\right)$. The value is slightly larger than the values found in the fits to the charged particle spectra in the central rapidity region, $n=7.1 \pm 0.2$ [1] [], $n=7.03 \pm 0.07$ [2]] (for $p_{\perp}>2 \mathrm{GeV} / \mathrm{c}$ ) and $n=7.25 \pm 0.03$ [3] (for $p_{\perp}>1.2 \mathrm{GeV} / \mathrm{c}$, with $\left.p_{\perp 0}=0.54 \mathrm{GeV} / \mathrm{c}\right)$.

The measured $p_{\perp}$ distribution in Fig. 3 can also be described by an exponential distribution of the form

$$
\frac{d^{2} \sigma}{d p_{\perp}^{2} d y}=a \cdot \exp \left(-b \cdot \sqrt{p_{\perp}^{2} c^{2}+m_{\pi^{0}}^{2} c^{4}}\right) .
$$

The fit to the data in the range $0.3 \leq p_{\perp} \leq 1.6 \mathrm{GeV} / \mathrm{c}$ is shown in Fig. $4 \mathrm{a}$ and yields the value $b=(5.7 \pm 0.3) \mathrm{GeV}^{-1}\left(\chi^{2} / \mathrm{ndf}=1.0\right)$. Such slope values are typical of soft hadronic interactions and expected in thermodynamical models (see e.g. [22]). Above $p_{\perp} \approx 1.5 \mathrm{GeV} / \mathrm{c}$, however, the exponential form tends to fall below the data, in contrast to the power law ansatz. This observation can be interpreted as an indication of the onset of hard parton-parton scattering processes which lead to a hardening of the transverse momentum distribution.

This interpretation is supported by a comparison of the data with the MC models. Fig. \#b shows again the cross section as a function of $p_{\perp}$, this time together with the model predictions of PYTHIA and PHOJET. The agreement between the data and the PYTHIA prediction is in general slightly better than for PHOJET. Also shown is the PYTHIA prediction for the amount of "direct" photon interactions. The contribution of this direct component accounts for $\sim 5 \%$ of the cross section at the lowest $p_{\perp}$ values, increasing up to $\sim 20 \%$ at the highest $p_{\perp}$ values accessed. Thus, in the rapidity and transverse momentum range covered by these measurements, the cross section is dominated by "resolved" photon processes.

The generator PYTHIA has also been used to search for evidence for a non-zero intrinsic transverse momentum, $k_{\perp}$, of partons inside the photon. As mentioned in section 3, PYTHIA offers the choice of several distributions for the parameter $k_{\perp}$ : a Gaussian, an exponential, and a power law ansatz. All three parametrizations have been used and the parameter $k_{\perp 0}$, which

\footnotetext{
${ }^{3}$ In Ref. [1] the error on the power $n$ is erroneously quoted as 2.0.
} 
defines their widths, varied between 0 and $2 \mathrm{GeV} / \mathrm{c}$. The largest influence on the predicted shape of the $y$ distribution appears in the highest $p_{\perp}$ interval. This interval, together with the curves predicted by PYTHIA for the Gaussian, exponential, and power law parametrizations, is shown in Fig. 5. We note that for these plots no additional cutoff for the primordial $k_{\perp}$ has been applied (see section 3). For all three functions large effects are seen when varying the parameter $k_{\perp 0}$, and low values of $k_{\perp 0}$ are preferred. Even a vanishing $k_{\perp 0}$ is consistent with the data.

Finally the behaviour of the distribution of the Feynman variable $x_{\mathrm{F}}$ has been investigated, with $x_{\mathrm{F}}$ defined as the fraction $p_{L} / p_{L}^{\max }$, where $p_{L}$ is the pion's momentum component parallel to the beam axis in the $\gamma p \mathrm{CM}$ frame, and $p_{L}^{\max }$ its maximum value in a given event, namely $p_{L}^{\max } \approx \sqrt{s_{\gamma p}} / 2$.

In Fig. 6, the differential cross section $d \sigma_{\gamma p} / d x_{\mathrm{F}}$ is displayed, obtained in the rapidity range $-3.5 \leq y \leq-1.5$. There is good agreement with the model predictions of PYTHIA and PHOJET. The cross section values are given in Table 3 .

\begin{tabular}{|c||c|c|c|c|c|c|}
\hline \multicolumn{1}{|c||}{} & \multicolumn{7}{c|}{$x_{\mathrm{F}}$} \\
\cline { 2 - 7 }$y$ & {$[0.1,0.15]$} & {$[0.15,0.2]$} & {$[0.2,0.25]$} & {$[0.25,0.3]$} & {$[0.3,0.4]$} & {$[0.4,0.6]$} \\
& $(0.125)$ & $(0.175)$ & $(0.225)$ & $(0.275)$ & $(0.348)$ & $(0.493)$ \\
\hline \hline$[-3.5,-1.5]$ & $477 \pm 67$ & $279 \pm 43$ & $209 \pm 35$ & $131 \pm 19$ & $65 \pm 12$ & $19 \pm 5$ \\
\hline
\end{tabular}

Table 3: Inclusive $\pi^{0}$ photoproduction cross sections $d \sigma_{\gamma p} / d x_{\mathrm{F}}(\mu \mathrm{b})$ in intervals of $x_{\mathrm{F}}$, in the rapidity range $-3.5 \leq y \leq-1.5$. The bin centres are given in addition to the interval limits.

Data from the Omega Photon Collaboration (WA69, Apsimon et al., [5]) are also shown in Fig. 6. They were obtained in fixed target $\gamma p$ collisions with an average photon energy of $80 \mathrm{GeV}$ (corresponding to $\sqrt{s_{\gamma p}}=12.3 \mathrm{GeV}$ ). From this $\mathrm{CM}$ energy and the $\pi^{0}$ mass one derives a CM rapidity range of up to 4.5 units available for neutral pions. These cross sections of inclusive $\pi^{0}$ photoproduction, available in Ref. [24] as $E d^{3} \sigma / d p^{3}$ in bins of $x_{\mathrm{F}}$ and $p_{\perp}$, have here been converted to cross sections differential in $x_{\mathrm{F}}$, by integrating over $p_{\perp}$ in each binf of $x_{\mathrm{F}}$. The two experiments are in good agreement, although small differences are seen in shape and normalization. These differences can be qualitatively understood from the difference in rapidity range; the cuts $-3.5 \leq y \leq-1.5$ (corresponding to $-5.5 \lesssim y \lesssim-3.5$ in the $\gamma p \mathrm{CM}$ ) reduce the $\mathrm{H} 1 \pi^{0}$ cross section at both large and small $x_{\mathrm{F}}$, a reduction which is only partly compensated for by the expected increase due to the rise of the total cross section between the two $\mathrm{CM}$ energies (factor $\sim 1.3$ [25]).

\section{Summary and Conclusions}

Inclusive $\pi^{0}$ photoproduction in the photon hemisphere has been analysed at HERA in the $\gamma p$ CM energy range $177<\sqrt{s_{\gamma p}}<242 \mathrm{GeV}$, with average $\sim 208 \mathrm{GeV}$. Differential cross sections with respect to transverse momentum $p_{\perp}$, rapidity $y$ and the Feynman variable $x_{\mathrm{F}}$ are presented.

\footnotetext{
${ }^{4}$ In the lowest bin of $p_{\perp}$ and $x_{\mathrm{F}}$, where a measurement with $80 \mathrm{GeV}$ beam energy is missing in [5], the corresponding measurement from the data set with average beam energy $140 \mathrm{GeV}$ has been used.
} 
The neutral pion data extend the measurements towards the previously unexplored domain of large rapidity in the photon fragmentation region, dominated by resolved $\gamma p$ interactions. This work extends previous $\mathrm{H} 1$ measurements performed with charged particles. In the phase space region covered by both analyses there is good agreement between the neutral and charged pion differential cross sections.

The differential cross section as a function of transverse momentum $p_{\perp}$ shows an exponential fall at lower $p_{\perp}$ values as seen in soft hadron-hadron collisions, but exhibits at values of $p_{\perp}$ larger than $1.5 \mathrm{GeV} / \mathrm{c}$ an enhancement which is expected for hard parton-parton scattering processes. The distribution in the entire $p_{\perp}$ range covered here is well described by a power law ansatz. The event generators PHOJET and PYTHIA are able to describe the measured cross sections in this kinematical domain, with a slight preference for PYTHIA. In the context of the PYTHIA model, the data are inconsistent with large values of an intrinsic transverse momentum in the photon.

\section{Acknowledgements}

We are grateful to the HERA machine group whose outstanding efforts have made and continue to make this experiment possible. We thank the engineers and technicians for their work constructing and maintaining the $\mathrm{H} 1$ detector, our funding agencies for financial support, the DESY technical staff for continuous assistance, and the DESY Directorate for the hospitality which they extend to the non-DESY members of the collaboration. A helpful communication with R. Engel is gratefully acknowledged.

\section{References}

[1] H1 Collab., I. Abt et al., Phys. Lett. 328B (1994) 176.

[2] H1 Collab., C. Adloff et al., Eur. Phys. J. C10 (1999) 363.

[3] ZEUS Collab., M. Derrick et al., Z. Phys. C67 (1995) 227.

[4] Ch. Berger et al., Phys. Lett. 47B (1973) 377;

D. P. Barber et al., Phys. Lett. 77B (1978) 233;

R.N. Diamond et al., Phys. Rev. D25 (1982) 41;

EMC Collab., J.J. Aubert et al., Z. Phys. C18 (1983) 189;

EHS-RCBC Collab., J.L. Bailly et al., Z. Phys. C22 (1984) 119;

NA14 Collab., E.Auge et al., Phys. Lett. 168B (1986) 163;

NA27 LEBC/EHS Collab., M. Aguilar-Benitez et al., Z. Phys. C34 (1987) 419;

NA22 Collab., I.V. Ajinenko et al., Z. Phys. C35 (1987) 7;

NA22 Collab., M.R. Atayan et al., Z. Phys. C54 (1992) 247.

[5] Omega Photon Collab., R.J. Apsimon et al., Z. Phys. C52 (1991) 397. 
[6] F.W. Büsser et al., Phys. Lett. 46B (1973) 471;

F.W. Büsser et al., Nucl. Phys. B106 (1976) 1;

C. Kourkoumelis et al., Z. Phys C5 (1980) 95;

UA2 Collab., M. Banner et al., Z. Phys. C27 (1985) 329.

[7] H1 Collab., I. Abt et al., Nucl. Instrum. Methods A386 (1997) 310 and 348.

[8] H1 SpaCal Group, T. Nicholls et al., Nucl. Instrum. Methods A374 (1996) 149;

H1 SpaCal Group, R.D. Appuhn et al., Nucl. Instrum. Methods A382 (1996) 395;

H1 SpaCal Group, R.D. Appuhn et al., Nucl. Instrum. Methods A386 (1997) 397.

[9] Martin Swart, Dr. rer. nat. dissertation, Universität Heidelberg, in preparation.

[10] H1 Calorimeter Group, B. Andrieu et al., Nucl. Instrum. Methods A336 (1993) 460 and 499.

[11] H1 Calorimeter Group, B. Andrieu et al., Nucl. Instrum. Methods A350 (1994) 57.

[12] R. Engel, PHOJET Manual (Version 1.05), Univ. Siegen Preprint 95-05 (1995) (revised Feb. 1997);

R. Engel, Z. Phys. C66 (1995) 203;

R. Engel and J. Ranft, Phys. Rev. D54 (1996) 4244.

[13] PYTHIA Version 5.722, T. Sjöstrand, Comp. Phys. Comm. 82 (1994) 74;

T. Sjöstrand, "PYTHIA 5.7 and JETSET 7.4", CERN-TH.7112/93 (1993) (revised Feb. 1994).

[14] H1 Collab., C. Adloff et al., Eur. Phys. J. C1 (1998) 97.

[15] B. Andersson, G. Gustafson and T. Sjöstrand, Phys. Lett. 94B (1980) 211;

B. Andersson et al., Phys. Rep. 97 (1983) 31.

[16] T. Sjöstrand and M. Bengtsson, Comp. Phys. Comm. 43 (1987) 367;

H. - U. Bengtsson and T. Sjöstrand, Comp. Phys. Comm. 46 (1987) 43;

T. Sjöstrand, CERN-TH-6488 (1992).

[17] A. Capella et al., Phys. Rep. 236 (1994) 225.

[18] G. A. Schuler and T. Sjöstrand, Nucl. Phys. B407 (1993) 539.

[19] M. Glück, E. Reya and A. Vogt, Phys. Rev. D46 (1992) 1973;

M. Glück, E. Reya and A. Vogt, Z. Phys. C53 (1992) 127.

[20] C. F. Weizsäcker, Z. Phys. 88 (1934) 612;

E. J. Williams, Phys. Rev. 45 (1934) 729;

S. Frixione, M. Mangano, P. Nason and G. Ridolfi, Phys. Lett. 319 B (1993) 339.

[21] R. Blankenbecler, S. J. Brodsky and J. F. Gunion, Phys. Rev. D18 (1978) 900.

[22] R. Hagedorn, Riv. Nuovo Cim. 6:10 (1983) 1.

[23] W.M. Geist et al., Phys. Rep. 197 (1990) 263. 
[24] HEPDATA, The Durham RAL Databases, http://durpdg.dur.ac.uk/HEPDATA

[25] The Particle Data Group, C. Caso et al., Eur. Phys. J. C3 (1998) 1. 

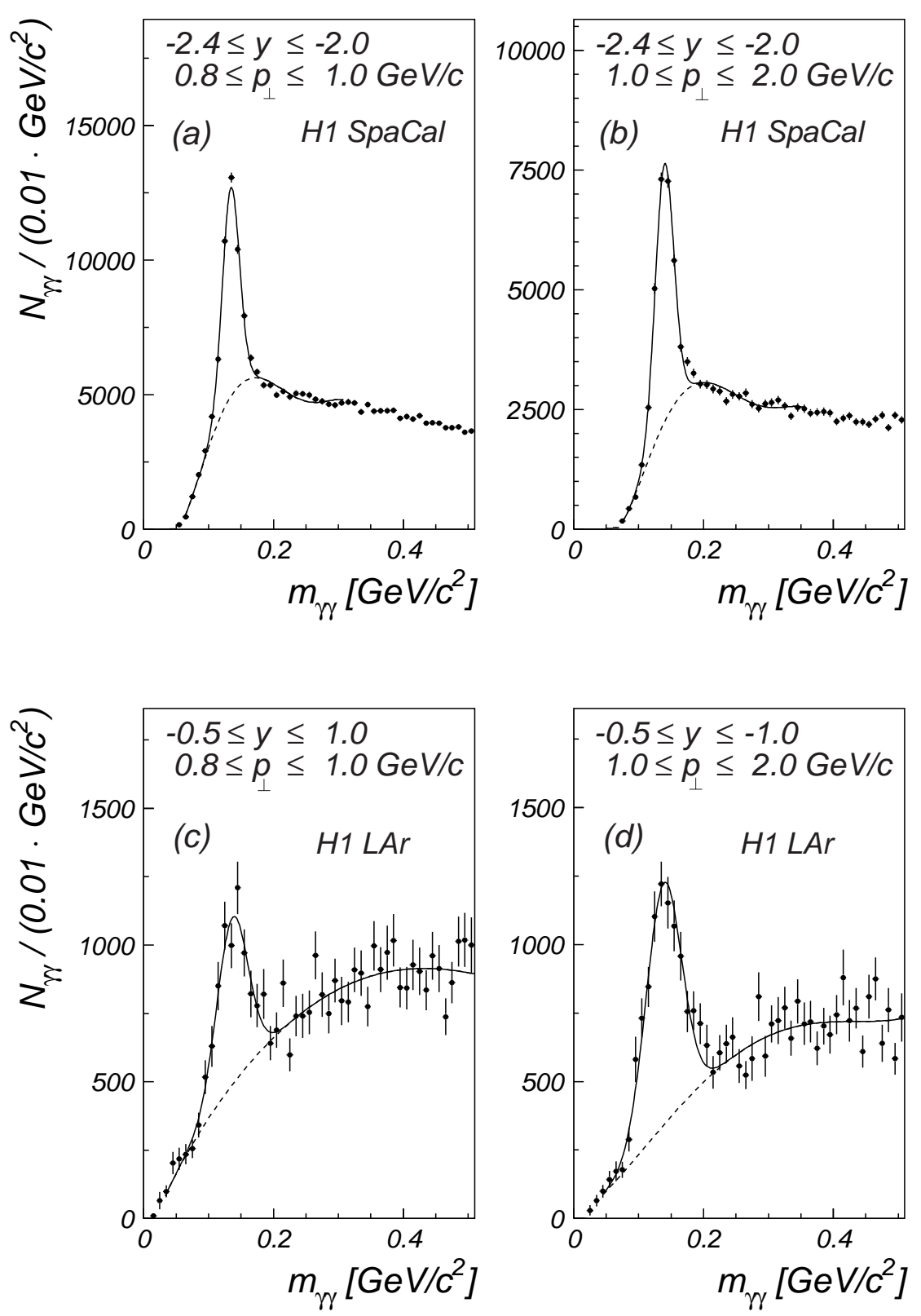

Figure 1: Two-photon invariant mass distributions reconstructed in the SpaCal calorimeter (a, b) and in the Liquid Argon calorimeter (c, d). The full curves show fits of a sum of a Gaussian and a background distribution (dashed curves) as described in the text. 


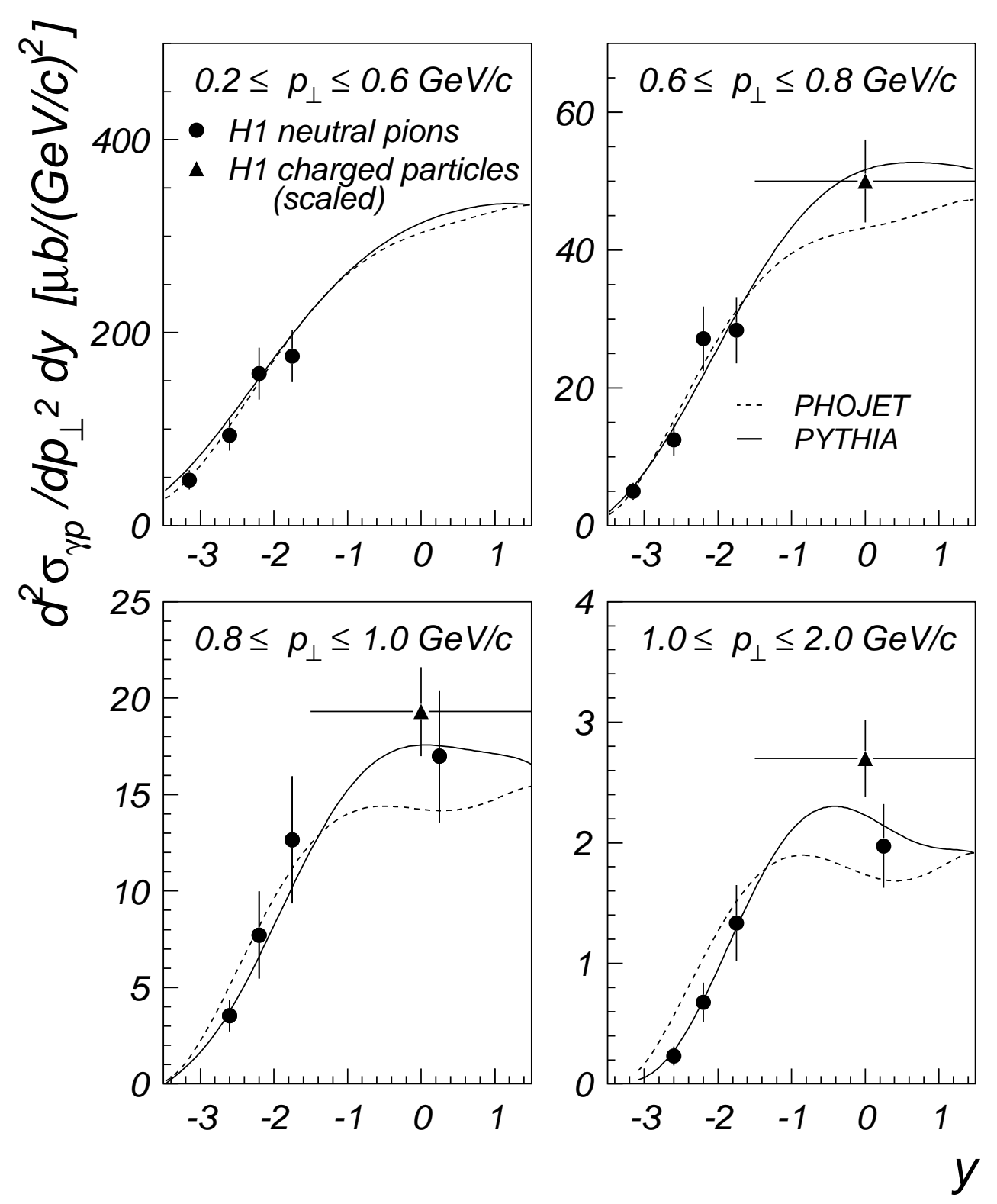

Figure 2: Inclusive $\pi^{0}$ photoproduction cross section as a function of laboratory rapidity $y$ in intervals of transverse momentum $p_{\perp}$ (full circles). The triangles are the corresponding cross section values for charged pions, derived from [1] by subtracting a fraction of $17.5 \%$ to account for the "non-pion" contribution, and then dividing by the isospin factor 2 (see text). The curves are the predictions of the PHOJET (dashed) and PYTHIA (full) event generators for neutral pions. 


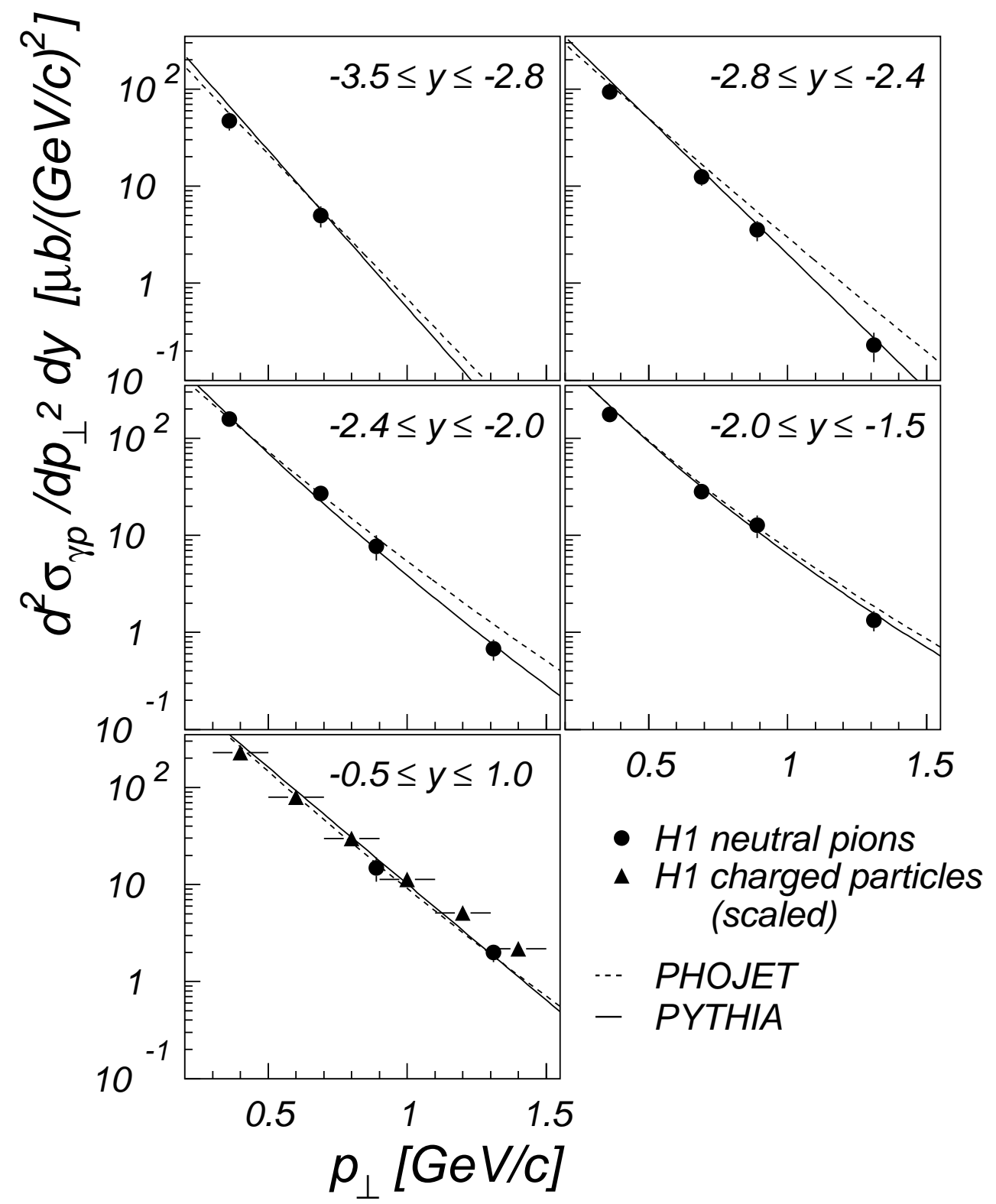

Figure 3: Inclusive $\pi^{0}$ photoproduction cross section as a function of transverse momentum $p_{\perp}$ in intervals of laboratory rapidity $y$ (full circles). The triangles are the corresponding cross section values for charged pions in the pseudorapidity range $|\eta| \leq 1.5$, derived from [1] by subtracting a fraction of $17.5 \%$ to account for the "non-pion" contribution, and then dividing by the isospin factor 2 (see text). The curves are the predictions of the PHOJET (dashed) and PYTHIA (full) event generators for neutral pions. 

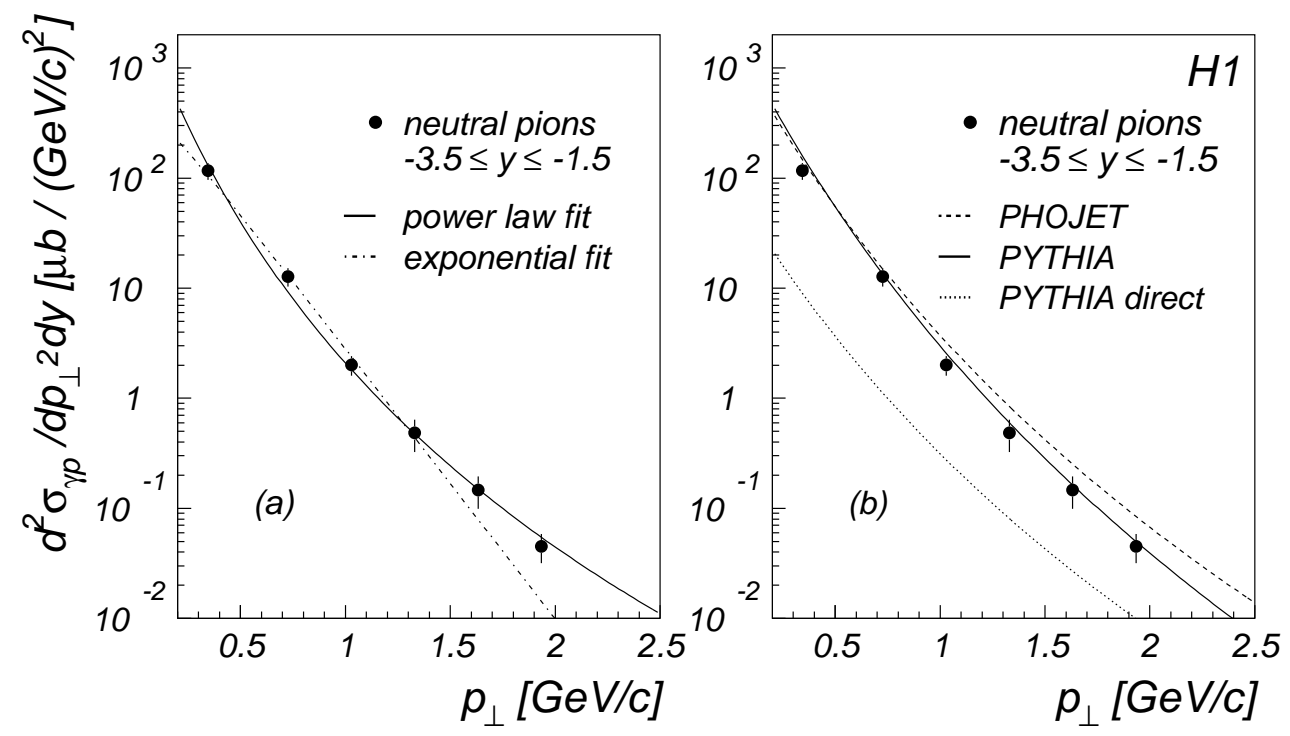

Figure 4: (a) Inclusive $\pi^{0}$ photoproduction cross section as a function of transverse momentum $p_{\perp}$ (circles), for the rapidity region $-3.5 \leq y \leq-1.5$. The dash - dotted curve is an exponential fit to the data in the range $0.3 \leq p_{\perp} \leq 1.6 \mathrm{GeV} / \mathrm{c}$; the full curve is the result of a power law fit to all data points (see text). (b) Same cross section as in (a) compared to predictions of PHOJET and PYTHIA. In addition, the contribution of direct photon interactions in PYTHIA is shown.


Figure 5: The $\pi^{0}$ cross section as a function of rapidity $y$ for the transverse momentum range $1.0 \leq p_{\perp} \leq 2.0 \mathrm{GeV} / \mathrm{c}$. The curves are the predictions of PYTHIA, labelled with the corresponding $k_{\perp 0}$ values, as described in the text. No additional cutoff on $k_{\perp}$ is applied (see text). 


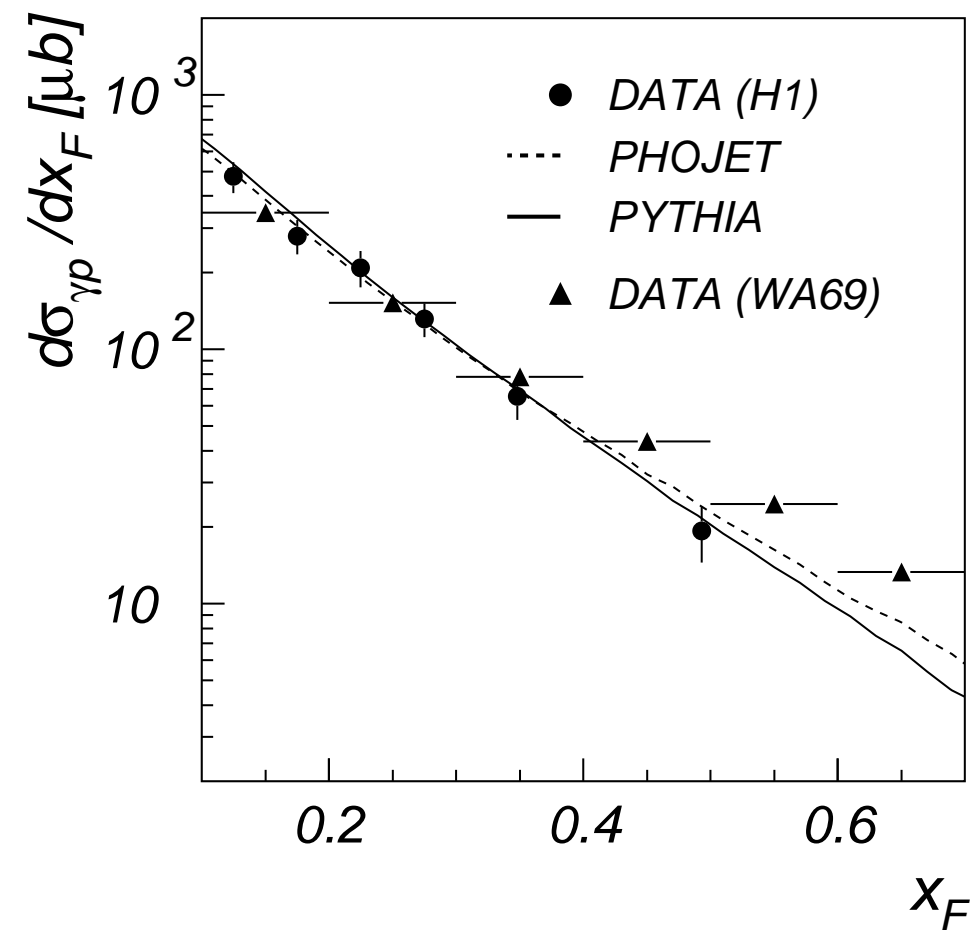

Figure 6: Inclusive $\pi^{0}$ photoproduction cross section as a function of Feynman $x$. The curves are the predictions of the PHOJET (dashed) and PYTHIA (full) models. The H1 data, at $\sqrt{s_{\gamma p}} \approx$ $208 \mathrm{GeV}$, as well as the MC model predictions, are obtained in the rapidity range $-3.5 \leq y \leq$ -1.5. The data of the Omega Photon Collaboration (WA69), at $\sqrt{s_{\gamma p}} \approx 12.3 \mathrm{GeV}$, were derived as described in the text. 